\title{
Adaptive Volumetric Texture Segmentation based on Gaussian Markov Random Fields
} Features

\author{
Yasseen Almakady ${ }^{\mathrm{a}, * *}$, Sasan Mahmoodi ${ }^{\mathrm{a}}$, Michael Bennett ${ }^{\mathrm{b}}$ \\ ${ }^{a}$ Faculty of Engineering and Physical Sciences, Electronics and Computer Science, University of Southampton, Highfield Campus, Southampton, SO17 1BJ, \\ United Kingdom \\ ${ }^{b}$ Southampton NIHR Respiratory and Critical Care Biomedical Research Centre, University Hospital Southampton NHS Foundation Trust, Southampton SO16 \\ 6YD, United Kingdom
}

\begin{abstract}
An adaptive method based on three dimensional Gaussian Markov Random fields (3D-GMRF) is proposed in this paper for volumetric texture segmentation. A feature vector is extracted for each voxel in a given volumetric texture image using an estimation cube. However, the selection of the size for this estimation cube causes some fundamental issues related to the uncertainty principle and the inability of the model to capture different texture patterns. These issues are tackled here by employing an adaptive method where the size of the estimation cube is adaptively varying to capture different patterns and also minimize the number of voxels that are related to different texture classes inside the estimation cube. The feature vectors that consist of the estimated parameters of the GMRF and form the parameter volume are hence employed to segment volumetric textures. These features are smoothed by applying an averaging filter using an adaptive averaging technique. Such an averaging filter improves the segmentation results considerably. Our method proposed here is evaluated on a synthetic volumetric texture dataset and compared with other methods to demonstrate the superiority of our segmentation method.
\end{abstract}

(c) 2020 Elsevier Ltd. All rights reserved.

\section{Introduction}

Texture analysis has received a great deal of attention due to its importance and application in computer vision and image processing. Many applications employ textures as an essential feature to deliver texture-based solutions in many fields such as those in biomedical imaging (Depeursinge et al., 2014). Most of the methods proposed for texture analysis have concentrated on acquiring texture features from two-dimensional images (2D). However, utilizing these methods to extract features from three-dimensional textures (3D) may not be appropriate for 3D textures (Almakady et al., 2018).

Texture is defined as 'the feel, appearance, or consistency of a surface or a substance' according to Oxford dictionaries. More specifically, Haralick (1979) describes texture images as 'the number and types of its primitives and the spatial organ-

\footnotetext{
${ }^{* *}$ Corresponding author:

e-mail: yham1n15@soton.ac.uk (Yasseen Almakady)
}

isation or layout of its primitives'. Finding a universal definition of texture is difficult, however, due to the diversity of natural and artificial texture images (Jain and Farrokhnia, 1990). The definition of texture proposed by Haralick (1979) can be slightly extended to describe the 3D texture as an arrangement of 3D texture primitives represented by a sequence of voxels. When dealing with 3D texture, the underlying texture can be divided into dynamic texture and volumetric texture (Maani et al., 2014). Dynamic texture (also referred to as space-time textures or temporal textures) is the texture represented by the spacetime $(x, y, t)$ and it is the accumulation of spatial texture defined in the $(x, y)$ domain over time $t$. Examples of dynamic texture include image sequences of $2 \mathrm{D}$ textures acquired at different times, such as videos of fire, sea waves, wavy trees, etc. Volumetric textures (or solid texture (Paulhac et al., 2009), on the other hand, refers to textures found in the 3D space defined by $(x, y, z) \in \mathbb{R}^{3}$. This type of texture is commonly considered in biomedical imaging fields in which 3D data acquisition techniques provide rich information about the internal structure of objects (Depeursinge et al., 2014). 
Textures are formed from small elements known as texels or texture primitive, which are defined as the local structure or the fundamental pattern of the texture (Petrou and Sevilla, 2006). Additionally, texture can also be described by textons, which are "the basic elements of pre-attentive perception." (Julesz, 1981). Later, Malik et al. (1999) provided an operational definition of textons for gray-level images by clustering outputs of an image convolved with a set of filters to produce prototypes. These prototypes are the textons which capture oriented bars, terminators, etc.

Analyzing textures aims at extracting texture-based features from images and applying these features to carry out different tasks like classification, synthesis, and segmentation. In this paper, a method based on three dimensional Gaussian Markov Random fields (3D GMRF) is proposed by using an adaptive approach to segment textures in volumetric images. The remainder of the paper is organized as follows: in Section 2, the existing volumetric segmentation methods are described. Our adaptive volumetric texture segmentation (AVTS) method is introduced in Section 3. In Section 4, the results are presented and discussed. The evaluation of our method on COPD detection is presented in Section 5 and finally Section 6 concludes the paper.

\section{Related work}

Image segmentation is the process of partitioning an image into a set of regions. Texture is one of the various features , along with colour and shape, that can be used for image segmentation. While many methods have been developed to segment textures in 2D images, little attention has been devoted to volumetric texture segmentation (Madabhushi et al., 2003). This is mainly due to computational challenges when an additional dimension is included (Aldasoro and Bhalerao, 2007). Extracting 2D textures from slices of volumetric images is a common approach but does not fully benefit from the valuable information contained in volumetric data (Depeursinge et al., 2014; Madabhushi et al., 2003). Most previous work on volumetric texture analysis has been focused on developing methods for medical image analysis. Based on Fourier domain filtering, a method for volumetric texture segmentation known as a Multiresolution Volumetric Texture Segmentation (M-VTS) is presented by Aldasoro and Bhalerao (2007). According to this method, texture measurements are extracted from the Fourier domain of the volumetric image via subband filtering using an orientation pyramid. The Bhattacharyya space is then used to select the most discriminant measurements and represents a feature space. The method uses a multiresolution classification algorithm to classify voxels before boundary refinement is performed. The disadvantage associated with this method is the involvement of a training stage which increases the computational cost of the method. Also, the method was not evaluated by using more than two-class textures. An automated segmentation method is proposed in Madabhushi et al. (2003) to segment prostatic adenocarcinoma in high-resolution Magnetic Resonance Imaging (MRI) data. In this method, the statistical, gradient, and Gabor filter features are computed at multiple scales and orientations capturing shape, size, and orientation of the tumor. However, this automatic method requires data to be manually labelled by experts which is not always available. In Akbari and Fei (2012), Wavelet-based support vector machines (W-SVMs) are trained to capture kidney texture in three-dimensional (3D) MR images to automatically segment kidneys. These texture features are integrated with geometrical data to differentiate the kidney from the surrounding tissues. Similarly, the kernel support vector machine (KSVM) is exploited in Zhan and Shen (2003) for automatic segmentation of the prostate in 3D ultrasound images. Based on a statistical shape model, this method extracts texture features at different scales and orientations using 2D Gabor filter banks applied to orthogonal planes, then (KSVM) is used to distinguish the prostate from surrounding tissues. The employment of SVM-based techniques requires parameter adjustment with more computational cost. Local binary patterns (LBP) are used in Abbasi and Tajeripour (2017) to extract texture features from orthogonal planes of 3D MRI images and utilized for the detection of the brain tumor.

\section{Adaptive Volumetric Texture Segmentation (AVTS)}

\subsection{D-GMRF Model}

GMRFs have been used to model image textures where model parameters are employed as features and used for texture classification (Dharmagunawardhana et al., 2016) and segmentation (Zhao et al., 2017; Courbot et al., 2018; Ates and Sunetci, 2019). In our work presented in Almakady et al. (2018) an extension to 3D-GMRF models is proposed where the estimated model parameters are successfully employed as features and used for volumetric texture classification.

Let $\Omega=\{v=(i, j, k) \mid 1 \leq i \leq H, 1 \leq j \leq W, 1 \leq k \leq D\}$ denote the set of grid points indexed by $(i, j, k)$ on a $H \times W \times D$ 3D lattice corresponding to voxels in a $3 \mathrm{D}$ image volume. The local conditional probability density function of the intensity value $g_{v}$ at location $v$ is defined by:

$p\left(g_{v} \mid y_{v+r}, r \in N_{v}\right)=\frac{1}{\sqrt{2 \pi \sigma^{2}}} \exp \left\{-\frac{1}{2 \sigma^{2}}\left(g_{v}-\lambda-\sum_{r \in N_{v}} \alpha_{r}\left(y_{v+r}-\lambda\right)\right)^{2}\right\}$

where $N_{v}$ is the neighborhood of the voxel at location $v, \alpha_{r}$ are the interaction parameters that measure the influence on a central voxel by its neighbors with intensity values of $y_{v+r}$ located at a relative position $r$ (Almakady et al., 2018; Petrou and Sevilla, 2006) and $\lambda$ is the mean of the voxels in the neighborhood $N_{v}$. In fact, coefficients $\alpha_{r}$ determine or measure the dependencies between the central voxel and other voxels in its neighborhood to demonstrate the structure of the texture in that neighborhood. The neighborhood scheme adopted here is a cube with size $n \times n \times n$ which is used to define parameters in equation (1). The probability distribution function presented in (1) is fitted to texture data by estimating the unknown parameters $\alpha_{r}, \sigma^{2}$ and $\lambda$. The Maximum Likelihood Estimation (MLE) is utilized here to estimate the parameters $\alpha_{r}$ and $\sigma^{2}$ (Genschel and Meeker, 2010) whilst $\lambda$ is calculated directly by taking the mean of the processed region to avoid dealing with non-linear equations which are expensive to solve and could suffer from 
instability (Almakady et al., 2018). Parameters estimation is performed by taking the partial derivative of the log-likelihood function with respect to $\alpha_{r}, \sigma^{2}$ and setting them to zero. This therefore leads to the following solutions:

$$
\begin{gathered}
\boldsymbol{\alpha}_{v}=\left(\sum_{v \in \Omega_{v}} \boldsymbol{y}_{v} \boldsymbol{y}_{v}^{T}+c^{2} I\right)^{-1}\left(\sum_{v \in \Omega_{v}} \boldsymbol{y}_{v} g_{v}\right) \\
\sigma_{v}^{2}=\frac{1}{\left|\Omega_{v}\right|} \sum_{v \in \Omega_{v}}\left(g_{v}-\boldsymbol{\alpha}_{v} \boldsymbol{y}_{v}\right)^{2}
\end{gathered}
$$

Where $\boldsymbol{\alpha}_{v}=\operatorname{row}\left[\alpha_{r}\right]_{1 \times M}$ and $\boldsymbol{y}_{v}=\operatorname{col}\left[y_{v+r}\right]_{M \times 1}$ for $r \in N, M$ is the number of voxels interacting with the central voxel, $T$ is the transpose operation, and the sum of $\boldsymbol{y}_{v} \boldsymbol{y}_{v}^{T}$ (i.e., neighbours of $g_{v}$ ) is taken over the estimation cube $\Omega_{v} \subset \Omega, I$ is an identity matrix and $c$ is a regularization parameter to avoid singularity in matrix inversion (Bjorkstrom, 2001). The estimation cube $\Omega_{v}$ is utilized to perform the estimation at each voxel. This is accomplished by collecting observations used for parameter estimation from a small region surrounding the voxel $v$. In details, instead of sliding the neighborhood cube, used to collect samples, inside the entire volume; the overlapping neighborhood cube is slid inside a smaller volume known here as estimation cube $\Omega_{v}$ with size $w \times w \times w$ centered at each voxel $v$ (see Figure 1 ) (Almakady et al., 2018). Sliding is performed voxel by voxel on all three axes covering $x, y$, and $z$ directions respectively. The estimation cube is also sliding inside the entire volumetric texture voxel by voxel on all three axes to cover the entire volumetric image. In our method presented in Almakady et al. (2019) the size of $\Omega_{v}$ is empirically selected to be constant over the whole texture image, however, a constant-size sometimes fails to capture relatively large texture patterns. Conversely, increasing the size of $\Omega_{v}$ may create issues with respect to the estimated parameters being over-smoothed. Such a phenomenon occurs when the estimation cube crosses a boundary and covers more than one class of textures, leading to blurred boundaries. Uncertainty principle is another reason for detected blurred boundaries. Theoretically speaking, to find the exact location of a certain point belonging to a boundary, the estimation volume needs to be as small as possible to locate the boundary point. However, a very small estimation volume can not capture the features of the textures surrounding the boundary point. To capture the texture features surrounding the boundary point, on the other hand, the estimation cube needs to be chosen large enough to cover at least one 3D texels for a reliable estimate. To tackle such issues, we propose an adaptive estimation cube based on a heuristic rule to select the optimal size of $\Omega_{v}$ before the estimation process is performed. We employed the statistical measure of entropy as a heuristic rule. It is observed that the entropy of a region containing more than one texture is more than or equal to the entropy of a region containing only one texture. Based on this measurement, a number of estimation cubes $\Omega_{v}^{i}$ i.e. $\Omega_{v}^{1}, \Omega_{v}^{2}, . ., \Omega_{v}^{i}$ with different sizes are centered at voxel $v$, then the entropy of each $\Omega_{v}^{i}$ is calculated. The optimal $\Omega_{v}$ is then selected by considering the $\Omega_{v}^{i}$ that has the least entropy as follow

$$
\Omega_{v}=\arg \min _{i}\left(H\left(\Omega_{v}^{i}\right)\right)
$$

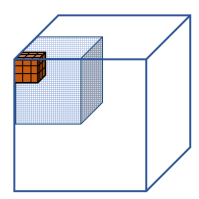

Figure 1. The neighborhood cube (small) and the estimation cube (medium) inside the entire (large) volumetric image.

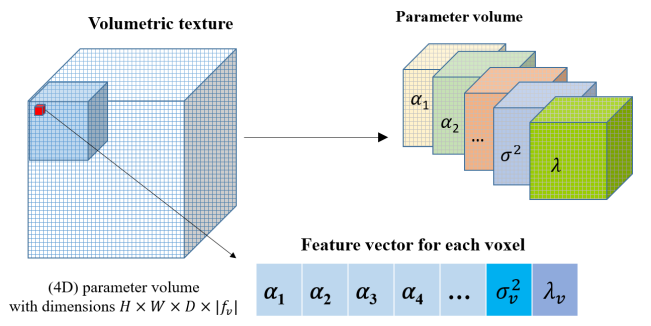

Figure 2. Graphical illustration of employing estimation cube to produce a feature vector for each voxel which results in parameters volumes.

Where $H$ is the entropy of each estimation cube defined as

$$
H\left(\Omega_{v}^{i}\right)=-\sum_{j} p_{j}^{i} \log p_{j}^{i}
$$

Where $p$ is the distribution of the intensity values in the estimation cube $\Omega_{v}^{i}$. If the entropies are equal, the smallest estimation cube is selected to minimize the number of voxels at boundaries that belong to different texture classes inside the estimation cube. Intuitively the smallest cube would improve our knowledge about the boundary location at the presence of various textures around the boundary. This procedure deals with the problems associated with boundary blurring by decreasing the size of $\Omega_{v}$ at boundaries and hence minimizing the number of voxels covered by the estimation cube which are related to different texture classes. It is noted that this process is not to localize boundaries but rather to determine whether a specific $\Omega_{v}^{i}$ contains more than one texture or not.

\subsection{Feature extraction}

The 3D GMRF model parameters $\boldsymbol{\alpha}_{v}, \sigma_{v}^{2}$, calculated in (23 ), and $\lambda_{v}$ are the components of the feature vector, to contain information about the local texture. The feature vector of voxel $v$ is given as:

$$
\boldsymbol{f}_{v}=\left[\boldsymbol{\alpha}_{v}, \sigma_{v}^{2}, \lambda_{v}\right]
$$

This feature vector is extracted for each voxel at location $v$. As the cubic neighborhood system is adopted in this paper with dimensions $n \times n \times n$ and $n=3$, therefore 26 voxels are involved in the neighborhood system which is equivalent in size to the estimated parameter vector $\boldsymbol{\alpha}_{v}$. Hence, in addition to $\sigma_{v}^{2}$ and $\lambda_{v}$, the size of the feature vector $f_{v}$ is $M+2$.

\subsection{Volumetric texture segmentation}

The texture features $f_{v}$ extracted in our proposed method AVTS are employed for segmentation. First, the 3D GMRF model parameters are calculated for each voxel by using the 
(a)
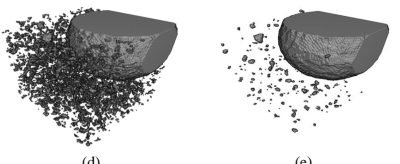

(e)

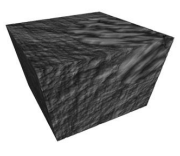

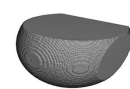

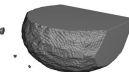

(f)
Figure 3. Illustration of averaging filter effect. (a) volumetric image. (b-c) ground truths. (d) segmented image based on GMRF model parameters without applying averaging filter. (e-f) segmented images after applying the averaging filter with different sizes to the parameter images.

proposed adaptive estimation cube to compose a feature vector $\boldsymbol{f}_{v}$. Since the feature vector is constructed at each voxel $v$ in a given volume; a four-dimensional (4D) parameter volume is produced with dimensions $H \times W \times D \times\left|\boldsymbol{f}_{v}\right|$ where $H, W, D$ are respectively the height, width, and depth of the volume (see Figure 2). Second, these parameter volumes are smoothed by sliding an averaging filter $V_{m}$ with dimensions $m \times m \times m$ inside the parameter volume to assign each $f_{v}$ new values. These new parameter values are the feature vectors filtered by $V_{m}$. This essential filtering step helps to eliminate isolated misclassified voxels, which are those voxels that have the same class label of surrounding neighbours, but they are misclassified with a different class label. The issue of isolated misclassified voxels arises either because of the presence of noise or due to the nature of texture structure in some regions of the image. Finally, the $k$-means clustering, with a manually adjusted $k$, is employed to cluster voxels into regions based on the volumetric texture features described by the feature vector $\boldsymbol{f}_{v}$. Figure 3 shows the effect of using the averaging filter $V_{m}$ to remove isolated misclassified voxels during the segmentation process. It can be observed that a greater number of incorrectly segmented voxels are eliminated when a larger $V_{m}$ is used to filter the feature space. Although a large $V_{m}$ is beneficial, particularly in the presence of noise, it performs poorly near boundaries. Accordingly, the adaptive averaging filter is proposed here to select an optimal size of the filter.

\subsection{Adaptive averaging filter}

Feature smoothing by applying an averaging filter is a proposed choice to reduce isolated misclassified pixels (Dharmagunawardhana et al., 2014; Chaudhuri and Sarkar, 1995; Hsiao and Sawchuk, 1989). Adjacent neighbours of a voxel are assumed to have the same texture class. However, noise can slightly affect the segmentation performance during $k$-means clustering, which results in isolated misclassified voxels. In our previous work presented in Almakady et al. (2019), the size of $V_{m}$ is considered constant over the whole volume. A large $V_{m}$ helps to remove some isolated misclassified voxels as well as the effect of noise during the segmentation process, but it also smooths boundaries between regions with different textures. The manual selection of the $V_{m}$ size limits the capability of the method to capture different texture structures. One way
Table 1. Mean entropy computed for every pair of homogeneous texture patches and their combination.

\begin{tabular}{|l|lll|}
\hline & Texture \#1 & Texture \#2 & Combined Textures \\
\hline Entropy (mean \pm std) & $5.98 \pm 1.48$ & $6.08 \pm 1.41$ & $\mathbf{6 . 5 3} \pm 1.24$ \\
\hline
\end{tabular}

to address this issue is to employ an adaptive approach according to which the value of $V_{m}$ varies adaptively according to a specific criterion. We exploit the valuable information encompassed in the 3D GMRF model parameters $\boldsymbol{\alpha}_{v}, \sigma_{v}^{2}$ to set up this criterion. In particular, the model parameter $\sigma_{v}^{2}$ represents a good criterion for $V_{m}$ selection. Given the parameter volume of $\sigma_{v}^{2}$, the elements collected by $V_{m}$ will have more considerable variation when $V_{m}$ contains boundaries due to the inclusion of deferent texture classes and also they will have lower variation when they belong to one texture class. Based on this observation, we propose our adaptive averaging filter depending on $\sigma_{v}^{2}$. To this end, multiple averaging filters $V_{m}^{i}$ (i.e. $V_{m}^{1}, V_{m}^{2}, . ., V_{m}^{i}$ ) with different sizes are placed at voxel $v$ in the parameter volume of $\sigma_{v}^{2}$, and then the variance is computed for each $V_{m}^{i}$. The averaging filter $V_{m}^{i}$ with the least variance is selected as follows.

$$
V_{m}=\arg \min _{i}\left(\operatorname{var}\left(V_{m}^{i}\right)\right)
$$

This is to ensure that the averaging filter has a maximum size in homogenous texture regions and a minimum size at the boundaries. Once the appropriate size is selected, the averaging process is applied before moving to the next location, as described in Section 3.3. It is essential to notice that the averaging filter is applied to parameter volume rather than to the given volumetric image. The details of AVTS are illustrated in Figure 4. There are some advantages for the selection of $\sigma_{v}^{2}$ over $\boldsymbol{\alpha}_{v}$ as a criterion for the adaptive averaging filter. In addition to its discrimination power, parameter $\sigma_{v}^{2}$ is a scalar for every voxel, making it easy to process it at multiple filter sizes.

\section{Method Evaluation}

The segmentation performance of our proposed method is evaluated using the RFAI database (Paulhac et al., 2009). This database contains different categories of volumetric textures for performance evaluation of the classification and segmentation. Synthetic volumetric texture samples with dimensions $128 \times 128 \times 128$ specified for segmentation evaluation are selected from this database. Three categories of images containing two, three, and four texture classes, respectively, are considered in this paper. Each category contains five texture images, for a total of 15 images. Figure 5 exhibits some examples of selected images containing two, three and four different textures with the corresponding ground truths. The features $\boldsymbol{f}_{v}=\left[\boldsymbol{\alpha}_{v}, \sigma_{v}^{2}, \lambda_{v}\right]$ are extracted at each voxel location $v$ using the proposed adaptive estimation cube. The neighborhood size is set by default to $n=3$. The regularization parameters $c$ (see Eq. 2) is empirically set to $c=100$ after testing for values 1,10 , 100 , and 1000. All previous settings are default, while the only parameter that requires a manual adjustment is $k$ of the $k$-means clustering algorithm which is manually adjusted to $\{2,3,4\}$ to cluster the voxels into $k$ separated volumes. During clustering, 


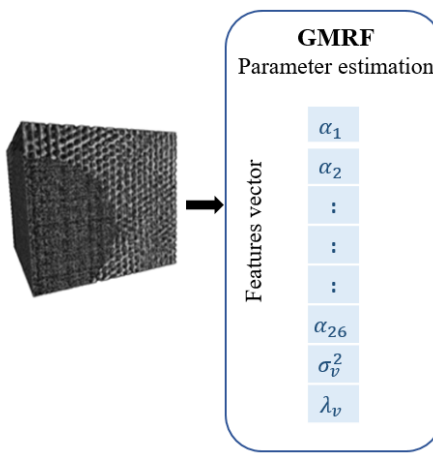

(a)

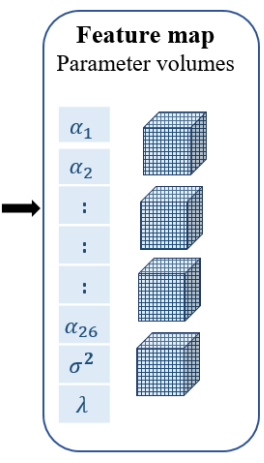

(c)

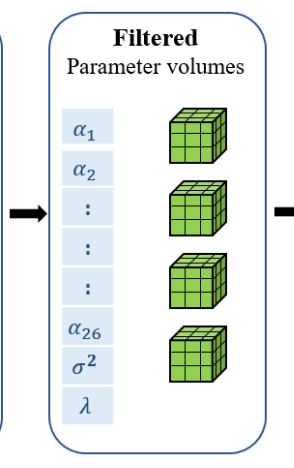

(d)

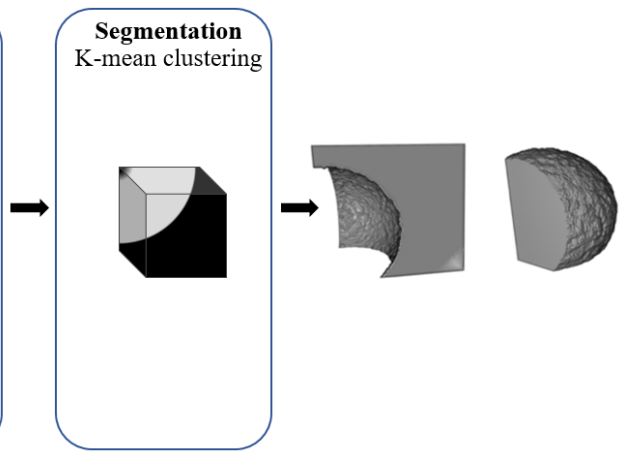

(e) (f)

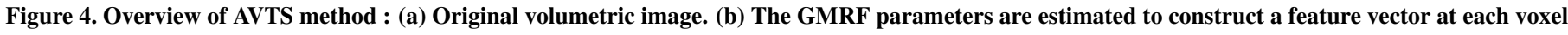

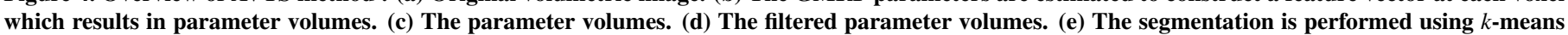
clustering algorithm. (f) Results.

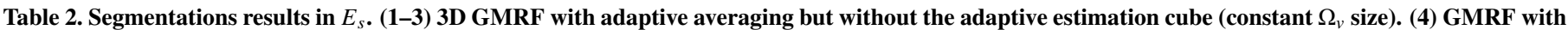
the adaptive estimation cube $\Omega_{v}$ but without adaptive averaging. (5) AVTS (with both the adaptive estimation cube and adaptive averaging).

\begin{tabular}{lllll}
\hline & Method & Two-class texture & Three-class texture & Four-class texture \\
\hline 1 & 3D GMRF, w=7 & $\mathbf{1 . 4} \pm \mathbf{0 . 8}$ & $16.3 \pm 8.7$ & $38.5 \pm 3.6$ \\
2 & 3D GMRF, w=9 & $1.6 \pm 1.1$ & $6.1 \pm 1.9$ & $33.51 \pm 4.7$ \\
3 & 3D GMRF, w=11 & $2.0 \pm 1.4$ & $\mathbf{6 . 0} \pm \mathbf{1 . 8}$ & $\mathbf{2 9 . 8} \pm \mathbf{3 . 3}$ \\
4 & 3D GMRF, adaptive $\Omega_{v}$ & $1.9 \pm 1.2$ & $12.1 \pm 9.4$ & $37.0 \pm 5.7$ \\
5 & AVTS & $1.8 \pm 0.9$ & $6.7 \pm 2.0$ & $33.4 \pm 3.5$ \\
\hline
\end{tabular}
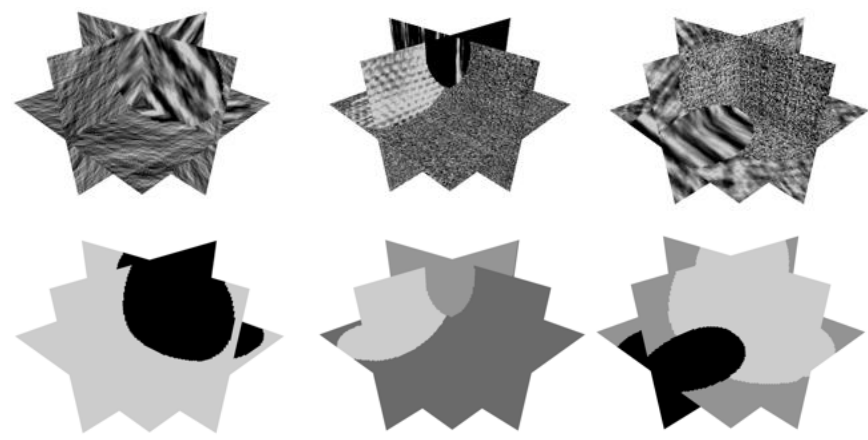

Figure 5. Examples of the internal structure of volumetric texture images with corresponding ground truths. Columns from left to right represents images with two, three and four texture classes respectively.

the maximum number of iterations is set to 1000 , whereas the clustering is performed only once. The segmentation performance is measured by the error rate $E_{s}$ and is calculated as the ratio of the number of incorrectly segmented voxels to the total number of voxels in the volume multiplied by 100 to turn it into a percentage. For each texture category, the results are obtained by computing the mean and standard deviation of $E_{s}$ values of all the images.

The first experiment presented here is to verify the hypothesis that the entropy can be used to measure the texture diversity. Four different volumetric texture datasets from the RFAI database are used in this experiment (i.e., Fourier, geometric, interpolated and mixed textures datasets). In this experiment, we initially measure the entropies of any two homogeneous texture patches in the dataset separately. Then we place one of the textures at the boundary of the other texture to form a new texture patch consisting of two homogeneous textures. We then measure the entropy of the new patch containing the two homogeneous textures. With the number of textures there is in the RFAI database, we can form 822 combinations of texture patches containing two homogeneous textures. The result of this experiment presented in Table 1, shows that $92.4 \%$ of all texture patches considered in this experiment have higher entropy when they contain two homogeneous textures. This is to say that the entropy of a single texture is less than when the entropy is measured for a patch containing two homogeneous textures. The aforementioned experiment demonstrates that with more than $92 \%$ confidence, using the entropy to select the size of the estimation cube, as explained in the next experiment, leads to a desirable segmentation performance among all texture samples.

In order to evaluate the segmentation performance of our method with respect to the adaptive estimation cube and averaging filter, two additional experiments are carried out: The first experiment aims to measure the efficacy of the adaptive estimation cube. In this experiment, the 3D GMRF parameters are estimated using a $\Omega_{v}$ with a constant size over the entire sample, and this estimation is compared with that of the AVTS method. We examine three different sizes of $\Omega_{v}$, i.e. $w=\{7,9,11\}$. The selection of estimation cube sizes is not restricted, and more sizes of the estimation cube can be tested. The averaging process is employed here for both methods, the 3D GMRF with a constant size of $\Omega_{v}$ and our adaptive method AVTS to focus on the effect of the adaptive $\Omega_{v}$. The second experiment aims at examining the effects of the adaptive averaging filter. In this 
experiment, the 3D GMRF parameters are estimated using the adaptive estimation cube without applying the averaging filter, and this method is compared with the AVTS method.

The segmentation results of our method in the experiments described above are presented in Table 2. The results reveal that employing an adaptive estimation cube during the estimation process combines consistent segmentation performance with the advantage of the automated selection of parameter $w$ among samples with different texture classes. Although the method without the adaptive estimation cube, especially $w=7$, performs well on the images with two-class textures, its performance declines when it is generalized to those with three or four texture classes, in which more boundaries exist. When $w=9$ is used, i.e., without any adaptive estimation cube, the method performs well among all samples except for textures with four classes. However, it still requires human intervention to select the value of $w$. The method with $w=11$ performs very well on the textures with three and four classes but demonstrates less segmentation performance with the two-class textures.

On the other hand, AVTS achieves consistent performance with competitive results in comparison with the other methods, whose parameter settings are selected manually. All methods demonstrate relatively less segmentation performance using four-class textures. This is because the higher the class textures inside an image, the more challenging the texture segmentation will become due to the fact that for a higher number of class textures, there are fewer voxels for parameter estimation (and therefore less accurate parameter estimation). Also, there are more boundaries among texture segments which increase the uncertainty between neighbouring texture segments. Possible solutions to improve the segmentation performance at the presence of many texture classes are: i) to combine other texture feature extraction methods such as LBP with our GMRF method to improve the segmentation performance and ii) to incorporate additional features such as colour or possibly edge information from textures to discriminate between texture classes at the boundaries.

Furthermore, the averaging filter is also essential to improve the performance of our segmentation method, especially if the optimal size of the averaging filter is selected, which is achieved through the adaptive averaging filter. Results in Table 2 rows (45) present the better performance of our segmentation method if the averaging filter is employed. The segmentation performance of our method AVTS is improved by employing both the adaptive estimation cube and the adaptive averaging filter Overall, AVTS has the advantage of an adaptive (automatic) scheme for adjusting the values of $V_{m}$ and $\Omega_{v}$. Some examples of the segmentation results obtained with AVTS are shown in Figure 8.

Additional experiments are conducted to evaluate the effect of the adaptive averaging filter in the presence of noise. Different levels of zero mean Gaussian noise proportional to signalto-noise ratio (SNR) with values $(100,90, \ldots, 10)$ are added to the volumetric texture samples with two texture classes. The SNR is calculated as a power ratio of the volumetric image over the noise. Next, AVTS is applied to the volumetric texture samples with added noise. Then this experiment is repeated with-

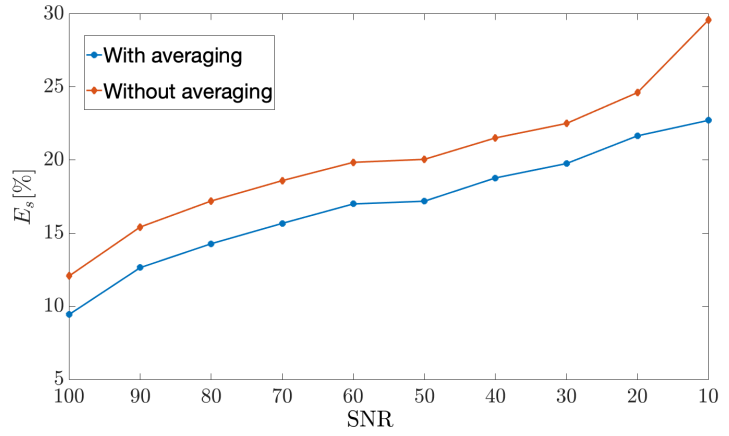

Figure 6. Segmentation error $E_{s}$ of AVTS with and without an averaging filter using volumetric texture samples with added noise.

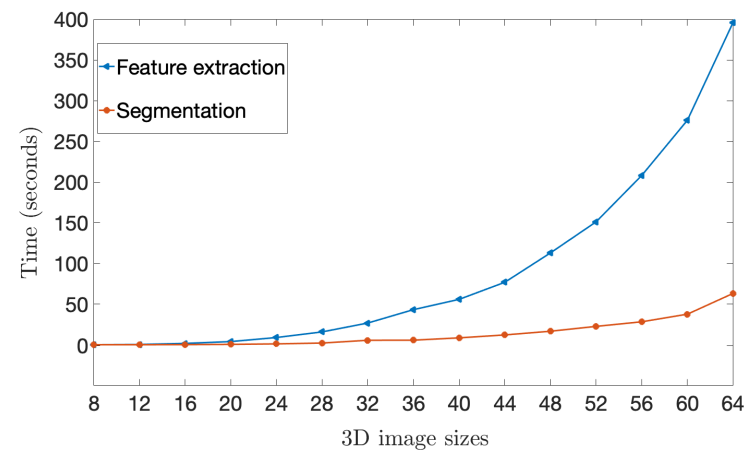

Figure 7. Computation time (in seconds) for the proposed method using various sizes the $3 \mathrm{D}$ image.

Table 3. Comparison results in $E_{s}[\%]$ of our method against M-VTS.

\begin{tabular}{lll}
\hline Datasets & \multicolumn{2}{c}{ Method } \\
\hline & M-VTS & AVTS \\
Gaussian Data & 6.2 & $\mathbf{0 . 9}$ \\
Oriented Data & 3.0 & $\mathbf{2 . 6}$ \\
\hline
\end{tabular}

out any adaptive average filtering. The results are presented as the mean of the segmentation error $E_{s}$ over all samples. It can be observed from Figure 6 that AVTS, which includes an adaptive averaging filter, has better segmentation performance than when the averaging filter is excluded.

To analyze the efficiency of AVTS, an experiment is conducted to examine the computation time. This experiment is implemented using a MATLAB R2020a environment running on Intel Core i5 $3.1 \mathrm{GHz}$ processor with $8 \mathrm{~GB}$ of RAM. Figure 7 depicts the computation time required by both the $3 \mathrm{D}$ GMRF feature extraction and segmentation processes. It can be observed that most of the time is consumed during the feature extraction stage because the 3D GMRF model is locally generated for each voxel to increase the discriminating power of the model. On the other hand, the computation time required by the segmentation is relatively low and is not highly influenced by the size of the $3 \mathrm{D}$ image.

\subsection{Comparison with other methods}

Our method is compared with M-VTS method (Aldasoro and Bhalerao, 2007) for volumetric texture segmentation. AVTS is implemented on the same dataset generated by Aldasoro and Bhalerao (2007) and compared with the previously reported M- 
Table 4. Comparison of Segmentations performance in $E_{s}[\%]$ for some existing segmentation methods reported in the literature with our proposed method.

\begin{tabular}{llll}
\hline Method & Tow-class texture & Three-class texture & Four-class texture \\
\hline$L B P_{26,1}^{\text {riu2 }}, N=7$ & $4.9 \pm 9.2$ & - & - \\
$L B P_{98,2}^{\text {riu2 }}, N=9$ & - & $7.3 \pm 5.4$ & - \\
$L B P_{98,2}^{\text {riu2 }}, N=7$ & - & - & $31.1 \pm 15.9$ \\
$D W T, d b 2, \beta=1, N=5$ & $4.5 \pm 6.0$ & - & - \\
$D W T, d b 2, \beta=2, N=5$ & - & $7.6 \pm 4.9$ & $21.8 \pm 6.1$ \\
$H U F, d b 2, \beta=2, N=5$ & $3.4 \pm 5.0$ & - & - \\
$H U F, d b 2, \beta=2, N=5$ & - & $9.0 \pm 3.7$ & - \\
$H U F, d b 2, \beta=1, N=9$ & - & - & $\mathbf{1 7 . 4} \pm \mathbf{1 2 . 1}$ \\
GLCM & $8.5 \pm 12.7$ & $14.5 \pm 6.0$ & $34.3 \pm 11.8$ \\
AVTS & $\mathbf{1 . 8} \pm \mathbf{0 . 9}$ & $\mathbf{6 . 7} \pm \mathbf{2 . 0}$ & $33.4 \pm 3.5$ \\
\hline
\end{tabular}

VTS results. The results of the comparison, presented in Table 3, demonstrate that for the Gaussian data, the segmentation error of AVTS is approximately six times lower than M-VTS, but for the oriented data, the improvement is only about $10 \%$.

Furthermore, our method is compared with a number of methods for which results using the RFAI database have been previously reported in the literature (Paulhac et al., 2015). These methods include the following: human-understandable features (HUF) (Paulhac et al., 2015), the 3D grey-level cooccurence matrix (3D GLCM) (Haralick, 1979; Haralick and Shanmugam, 1973), the 3D LBP method (Paulhac et al., 2008) and the 3D Discrete Wavelet Transform (3D DWT) (JafariKhouzani et al., 2004). The performance measurement used to evaluate these methods as reported in (Paulhac et al., 2015), is based on the generic discrepancy measure (Cardoso and CorteReal, 2005). The best results achieved by each method with its parameter setting are presented in Table 4 alongside the results obtained using our method. The results are presented by taking the mean and the standard deviation of the results obtained for all images in each category. Our method achieves good segmentation results compared with other methods using 3D images with two and three textures. Using four-class texture images, our method performs less or closer to the other methods. Nevertheless, AVTS is semi-automatic and does not require manual parameter setting (except for the number of textures, $k$ ) for segmentation. In contrast, the performances of the other methods investigated here depend on the manual adjustment of their parameters. Such a manual adjustment in some cases produces a high variance in the results.

\section{Application to COPD Detection}

In this experiment, we evaluate our method using a clinical dataset of lung images for the detection of chronic obstructive pulmonary disease (COPD). Clinically, COPD is considered as a progressive lung disease which is common, preventable, and treatable (Vogelmeier et al., 2017). In this disease, the patients suffer from persistent respiratory symptoms and airflow limitation that is due to airway and/or alveolar abnormalities (Vogelmeier et al., 2017). This disease is characterized by pathological changes affecting the texture of a healthy lung in the CT scan images of lungs. The changes in lung textures can be utilized to localize the regions of the lungs that are affected by COPD. An abnormal subject is selected from the clinical dataset of full-lung HRCT volumetric images with a size of $256 \times 256 \times 256$ and used in this experiment. Figure 9 shows
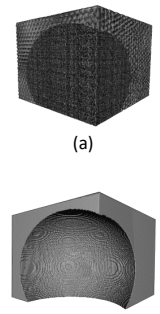

(d)

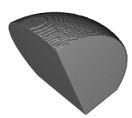

(g)

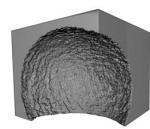

(j)

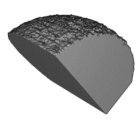

(m)

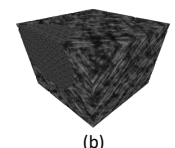

(b)
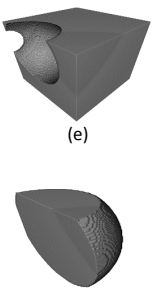

(h)
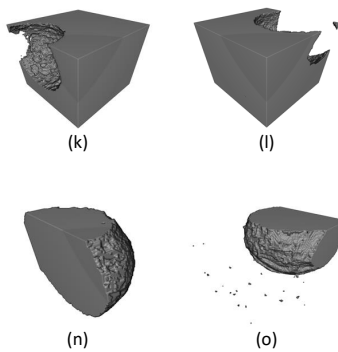

(o)
Figure 8. Examples of the segmentation results using our method: (a-c) the original volumetric images. (d-i) corresponding ground truths. (j-o) segmentation results.

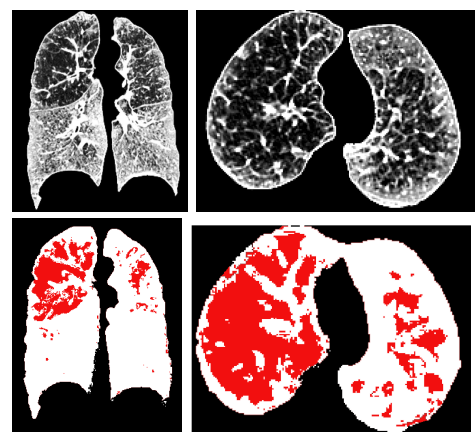

Figure 9. Examples of the segmentation results using our method on the clinical dataset : (Top) The original volumetric images of the lung from axial (left) and coronal (right) views . (Bottom) Segmentation results.

the segmentation results of selected slices from the axial and coronal views. In Figure 9 (top), the darker regions inside the lung CT- scans indicate the presence of COPD in the lung. 


\section{Conclusion}

In this paper, an adaptive method for volumetric texture segmentation, named AVTS, is introduced. Based on the 3D GMRF model, the estimated parameters are employed as a feature vector to describe the local textures. These features are extracted for each voxel in the volume using our proposed adaptive estimation cube. The feature extraction generates parameter volumes that are smoothed by an adaptive averaging filter, before the segmentation is performed, to remove isolated misclassified voxels and discriminate between regions with different textures. The results of the experiments conducted on volumetric textures involving different texture classes demonstrate the excellent performance of AVTS in extracting discriminative features and segmenting the volumetric textures. Additional experiments are also carried out to compare AVTS with existing methods from the literature, and the results demonstrate that our proposed method produces competitive results being a semiautomated method that does not require manual adjustment of the estimation cube and filter sizes.

The limitation of this method is the manual selection of $k$ in the $k$-means clustering algorithm that is used to specify the number of texture classes in a volumetric image. Hence, automating the choice of $k$ would be beneficial to eliminate the human intervention in this method, and it can be a direction for future work. Another avenue of future work could be minimizing the computation time required for parameter estimation. This could be achieved by improving the 3D GMRF parameter estimation. The last but not the least suggested future work could be measuring the segmentation performance of the method when non-linear filtering is used to filter the volumes of parameters before segmentation is performed.

\section{Acknowledgments}

Imaging data were acquired from a previous study conducted by the Southampton NIHR Biomedical Research Centre, which was approved by the Southampton and South West Hampshire local research ethics committee (LREC number: 09/H0502/91) and the University Hospital Southampton Foundation Trust Research and Development Department. The research in this paper is partially funded by (TVTC) in Saudi Arabia.

\section{References}

Abbasi, S., Tajeripour, F., 2017. Detection of brain tumor in 3d mri images using local binary patterns and histogram orientation gradient. Neurocomputing 219, 526-535

Akbari, H., Fei, B., 2012. Automatic 3d segmentation of the kidney in $\mathrm{mr}$ images using wavelet feature extraction and probability shape model, in: Medical Imaging 2012: Image Processing, International Society for Optics and Photonics. p. 83143D.

Aldasoro, C.C.R., Bhalerao, A., 2007. Volumetric texture segmentation by discriminant feature selection and multiresolution classification. IEEE Transactions on Medical Imaging 26, 1-14.

Almakady, Y., Mahmoodi, S., Conway, J., Bennett, M., 2018. Volumetric texture analysis based on three-dimensional gaussian markov random fields for copd detection, in: Annual Conference on Medical Image Understanding and Analysis, Springer. pp. 153-164.

Almakady, Y., Mahmoodi, S.M., Bennett, M., 2019. Gaussian markov random fields-based features for volumetric texture segmentation, in: 2019 IEEE Conference on Multimedia Information Processing and Retrieval (MIPR), IEEE. pp. 212-215.
Ates, H.F., Sunetci, S., 2019. Multi-hypothesis contextual modeling for semantic segmentation. Pattern Recognition Letters 117, 104-110.

Bjorkstrom, A., 2001. Ridge regression and inverse problems. Stockholm University, Department of Mathematics .

Cardoso, J.S., Corte-Real, L., 2005. Toward a generic evaluation of image segmentation. IEEE Transactions on Image Processing 14, 1773-1782.

Chaudhuri, B.B., Sarkar, N., 1995. Texture segmentation using fractal dimension. IEEE Transactions on pattern analysis and machine intelligence 17, $72-77$.

Courbot, J.B., Monfrini, E., Mazet, V., Collet, C., 2018. Oriented triplet markov fields. Pattern Recognition Letters 103, 16-22.

Depeursinge, A., Foncubierta-Rodriguez, A., Van De Ville, D., Müller, H., 2014. Three-dimensional solid texture analysis in biomedical imaging: review and opportunities. Medical image analysis 18, 176-196.

Dharmagunawardhana, C., Mahmoodi, S., Bennett, M., Niranjan, M., 2014. Gaussian markov random field based improved texture descriptor for image segmentation. Image and Vision Computing 32, 884-895.

Dharmagunawardhana, C., Mahmoodi, S., Bennett, M., Niranjan, M., 2016. Rotation invariant texture descriptors based on gaussian markov random fields for classification. Pattern Recognition Letters 69, 15-21.

Genschel, U., Meeker, W.Q., 2010. A comparison of maximum likelihood and median-rank regression for weibull estimation. Quality Engineering 22, 236-255.

Haralick, R.M., 1979. Statistical and structural approaches to texture. Proceedings of the IEEE 67, 786-804.

Haralick, R.M., Shanmugam, K., 1973. Textural features for image classification. IEEE Transactions on systems, man, and cybernetics , 610-621.

Hsiao, J.Y., Sawchuk, A.A., 1989. Supervised textured image segmentation using feature smoothing and probabilistic relaxation techniques. IEEE Transactions on pattern analysis and machine intelligence 11, 1279-1292.

Jafari-Khouzani, K., Soltanian-Zadeh, H., Elisevich, K., Patel, S., 2004. Comparison of $2 \mathrm{~d}$ and $3 \mathrm{~d}$ wavelet features for tle lateralization, in: Medical Imaging 2004: Physiology, Function, and Structure from Medical Images, International Society for Optics and Photonics. pp. 593-602.

Jain, A.K., Farrokhnia, F., 1990. Unsupervised texture segmentation using gabor filters, in: 1990 IEEE international conference on systems, man, and cybernetics conference proceedings, IEEE. pp. 14-19.

Julesz, B., 1981. Textons, the elements of texture perception, and their interactions. Nature 290, 91-97.

Maani, R., Kalra, S., Yang, Y.H., 2014. Robust volumetric texture classification of magnetic resonance images of the brain using local frequency descriptor. IEEE Transactions on Image Processing 23, 4625-4636.

Madabhushi, A., Feldman, M., Metaxas, D., Chute, D., Tomaszewski, J., 2003. A novel stochastic combination of $3 \mathrm{~d}$ texture features for automated segmentation of prostatic adenocarcinoma from high resolution mri, in: International Conference on Medical Image Computing and Computer-Assisted Intervention, Springer. pp. 581-591.

Malik, J., Belongie, S., Shi, J., Leung, T., 1999. Textons, contours and regions: Cue integration in image segmentation, in: Proceedings of the Seventh IEEE International Conference on Computer Vision, IEEE. pp. 918-925.

Paulhac, L., Makris, P., Ramel, J.Y., 2008. Comparison between 2d and 3d local binary pattern methods for characterisation of three-dimensional textures, in: International Conference Image Analysis and Recognition, Springer. pp. 670-679.

Paulhac, L., Makris, P., Ramel, J.Y., Gregoire, J.M., 2015. A framework of perceptual features for the characterisation of $3 \mathrm{~d}$ textured images. Signal, Image and Video Processing 9, 305-329.

Paulhac, L., Makris, P., Ramel, J.Y., et al., 2009. A solid texture database for segmentation and classification experiments., in: VISAPP (2), pp. 135-141.

Petrou, M., Sevilla, P.G., 2006. Image processing: dealing with texture. volume 1. Wiley Chichester.

Vogelmeier, C.F., Criner, G.J., Martinez, F.J., Anzueto, A., Barnes, P.J., Bourbeau, J., Celli, B.R., Chen, R., Decramer, M., Fabbri, L.M., 2017. Global strategy for the diagnosis, management and prevention of chronic obstructive lung disease 2017 report. Respirology 22, 575-601.

Zhan, Y., Shen, D., 2003. Automated segmentation of $3 d$ us prostate images using statistical texture-based matching method, in: International Conference on Medical Image Computing and Computer-Assisted Intervention, Springer. pp. 688-696.

Zhao, Q.h., Li, X.1., Li, Y., Zhao, X.m., 2017. A fuzzy clustering image segmentation algorithm based on hidden markov random field models and voronoi tessellation. Pattern Recognition Letters 85, 49-55. 